\title{
Design and Modelling of Wireless Sensor System
}

\author{
Ashwaq. Q. Hameed ${ }^{1}$, Majd. N. Abass ${ }^{2}$ \\ Technology University, College of Electrical Engineering, Baghdad, Iraq ${ }^{1}$ \\ University of Information Technology and Communication, Baghdad, Iraq $^{2}$
}

\begin{abstract}
A wireless sensor system is a system that provide and utilising any number of sensor modules, any number of communication devices, The sensor modules have sensors for sampling data, and wirelessly transmit data to their corresponding communication device, and receive transmission instructions from the same. This project present an introduction to wireless sensor system, Types of sensors and describe its working in general form. Next presents a design of wireless system for sensor temperature. The main purpose of this research is to design a model forreading and measuring temperature signals. The system reads data from sensor and then transfer it to the computer to be read in MATLAB program.
\end{abstract}

Keywords: Sensor, TMP36, XBee Module, Temperature Wireless Sensor.

\section{INTRODUCTION}

Communication system transfer information between different places, this information could be temperature, sound, pressure, movement and others [1].

A wireless sensors system are provide a single connect (one jump) to the user from the sensor.

A wireless sensors system and networks are represents a great intellectual challenge so there has been big academic interest in their expansion and behaviour.

It is sense and controls the environment, enabling interaction between users or computers and the surrounding environment [5].

The sensor device is the main parts of a wireless sensors system. The hardware of a sensor generally includes four parts: the power and power management module, a sensor, a microcontroller, and a wireless transceiver.

The wireless connection networks are managed through Radio waves system. AM $\backslash$ FM Radio, satellite Radio, satellite TV[2]. The Wireless technology many advantages which is facilitated life and make it convenient, it is also Freedom of placement, the system designs are easy, no/low maintenance, no battery replacement, and the range of wireless technology can be impressive. While the equipment you use may break (just as wired equipment) the signals themselves never break[3].

\section{SENSOR CHARACTERISTICS}

All sensors featuring distinctive characteristics describe their capabilities and potentials, the most important characteristics of sensors are:

1. Sensitivity: Any simple change in the input leads to a change in the output.

2. Response Time: the time required to do a change in the input will lead to a change in the output result.

3. Reliability: Accuracy of measurement to give reliable results.

4. Repeatability: ability to provide repeated measures.
5. Bandwidth: results with high resolution for transmitting signal [5][8].

\section{APPLICATION OF SENSORS DEVICES}

It is obvious to the user how important sensors and how its uses and applications are extent in public life, so we will review here some of these uses:

1- In a mercury-based glass thermometer, the input is temperature. The liquid extends and shrinks in response; Temperature makes the liquid rise or fall in the thermometer and this give us the readable measure.

2- An oxygen sensor in a car's emission control system detects the gasoline/oxygen ratio, usually through a chemical reaction that generates a voltage. A computer in the engine reads the voltage, if the mixture is not optimal, readjusts the balance.

3- Motion sensors in various systems including home security lights, automatic doors typically send out some type of energy, such as microwaves, ultrasonic waves or light beams and detect when the flow of energy is interrupted by something entering its path.

4- A photo sensor detects the presence of visible light, infrared transmission (IR), and/or ultraviolet (UV) energy.

\section{SENSORS CLASSIFICATION}

Sensors are classified based on the signal generated by these devices and these types are:

1. Active sensor: External sources of power are required (excitation voltage) which supply power to the output signal.

2. Passive sensor: The output power is almost fully provided by the measured signal without an excitation voltage.

3. Analog sensor: The sensor generated the signal continuous and proportional to the measured. 
Vol. 4, Issue 2, February 2017

4. Digital sensor: The signal generated or reflected by the sensor is binary.

5. Input Output configuration: Use whenever possible one of these method: method of inherent insensitivity, method of high gain feedback, method of calculated output corrections, method of signal filtering and method of opposing inputs [8].

\section{COMMON SENSING WAYS}

There are several ways to sense and show the results when the sensor detects a high or low temperature.

The output will be one of the following forms:

1- Analog output: the signal will be as a voltage.

2- Serial output: The signal will be in the form of a Readings series.

3- Logic output: The signal will be in $0 / 1$ or on/off.

4- Other ways used thermocouple, thermostat/Amplifiers which used comparators or programmable gain amplifier (PGA). [13]

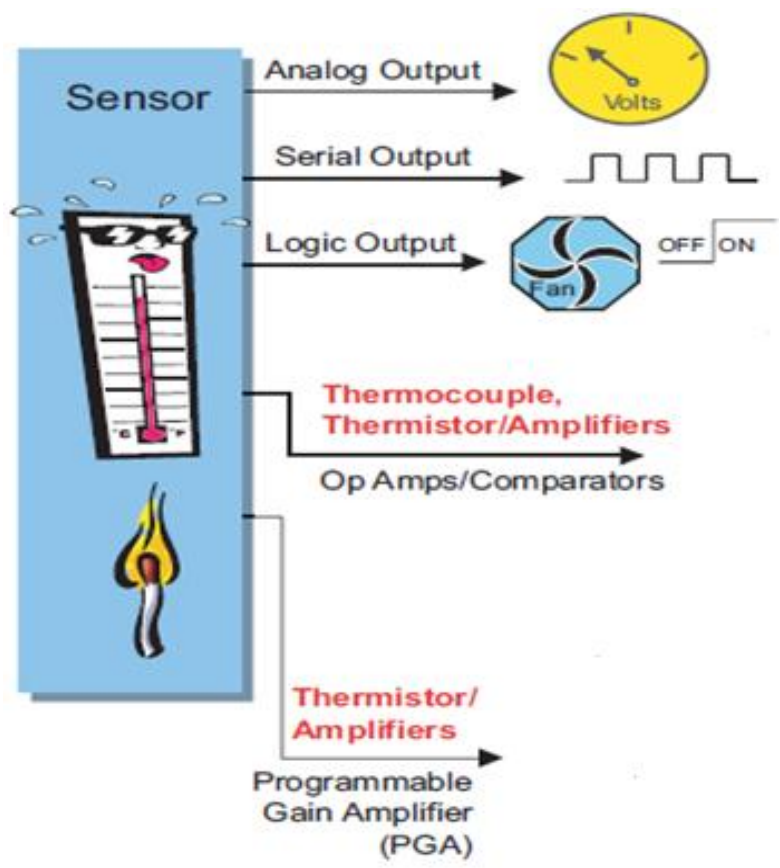

Fig. 1 output forms

\section{MODEL OF WIRELESS TEMPERATURE SYSTEM}

To design a wireless temperature system we need a number of devices and equipment that enables us to send, receive the signal wirelessly, read, monitored and then taken action depending on the reading signal. In this system we will need the following parts: temperature sensor that used to sense the room temperature, Laptop or computer, two XBees one of them keep in the room that we want to read its temperature connect it to the temperature sensor and the other one connect to the computer. In Figure 2 a simple design model for a Wireless Temperature System.

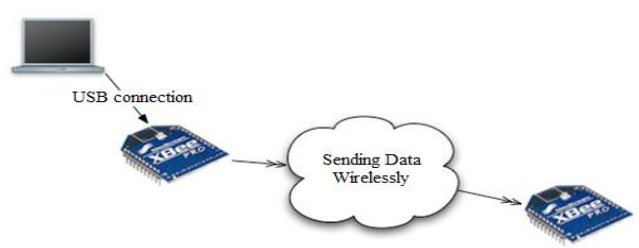

Fig.2 simple Wireless Temperature System

A. Temperature Sensor

Temperature Sensor:- it's a chip that tells you what the surroundings temperature is, A new technique are used in these sensors to determine the temperature which is name a solid-state. And this mean that they don't use the old method to determine the temperature (example mercury in old thermometers or a bimetallic strip in thermometers which used in home) and it doesn't use thermistors (temperature sensitive resistors). Instead of that, they use the fact of temperature increment, the voltage across a diode increases at a known rate (Technically, this is actually the voltage drop between the base and emitter.) By precisely amplifying the voltage change, it is easy to generate an analog signal that is directly proportional to temperature. There have been some improvements on the technique but, essentially that is how temperature is measured [15].

These sensors have no moving parts, they are accurate, does not wear out, do not need calibration, work under several conditions, and are consistent between sensors and readings. In addition they are very inexpensive, Temperature sensor I used for this project is the TMP36 [15]. Fig. 3 show the TMP36 temperature sensor.

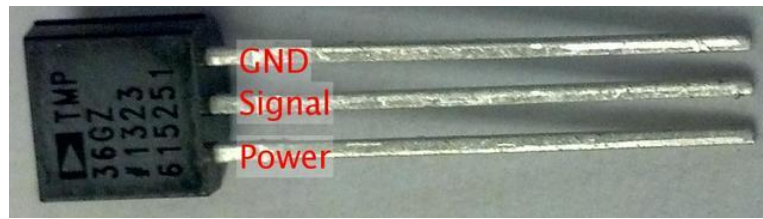

Fig.3 TMP36 Temperature Sensor

B. Measure of the Temperature

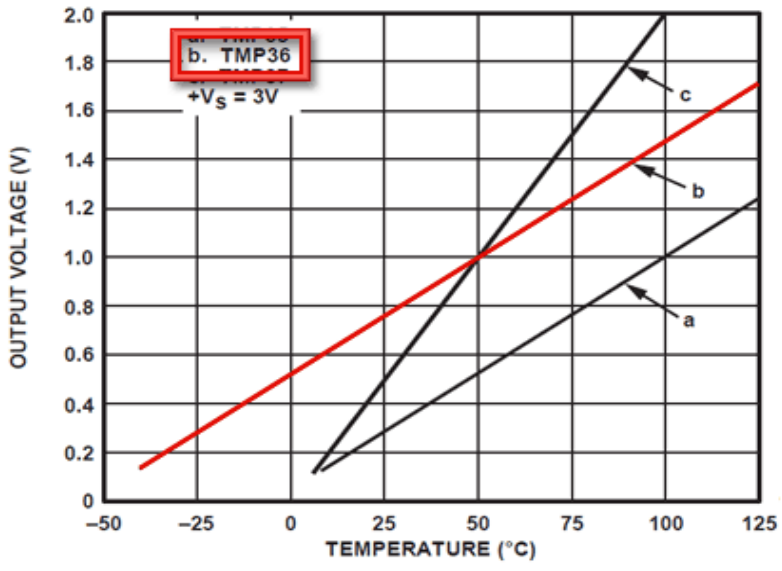

Fig.4 Output voltage vs. Temperature 
Vol. 4, Issue 2, February 2017

TMP36 has been used to measure the temperature by connect the left pin to power $(2.7-5.5 \mathrm{~V})$ and the right pin to ground, and the middle pin will have an analog voltage that is directly proportional (linear) to the temperature [15]. The analog voltage is independent of the power supply as show in figure 4 .

\section{XBee Series 2 Modules}

It is an electronic piece which can send and receive instructions, it has several types and the differences between these types are in energy consumption and transfer range. This module have small pins that cannot be connected directly with the panel BreadBoard test so we need to be installed on a dedicated circuit first, which is an XBee Shield, in figure 6 we see an XBee and its shield.

XBee Shield: This circuit has provided the possibility of adding XBee Module on the Arduino circuit where the Arduino play the role of mediator between the two.

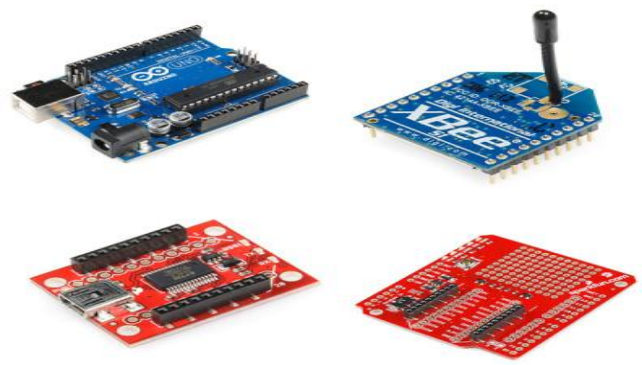

Fig. 6 XBee and XBee shield

\section{XBee Explorer USB}

This unit is simple and easy to use, it is work with all XBee modules including the type we are use, Plug the unit into the XBee, attach a mini USB cable, to access directly to the serial and programming pins on the XBee unit. In figure 7 we see the XBee Explorer USB. in addition we use a Mini-USB cable to connect the XBee to the USB unit.

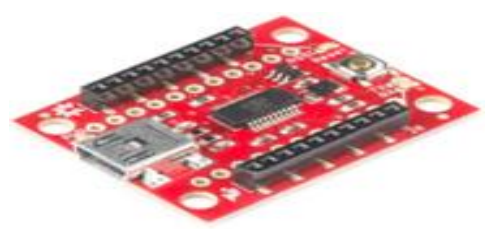

Fig. 7 The XBee Explorer USB

\section{THE WIRELESS TEMPERATURE SENSORS SYSTEM}

To design the wireless temperature sensor system we need to use the XBee modules, The Math Works MATLAB program, MATLAB is used to wirelessly control, read the voltage and to make interfacing between MATLAB and the XBee network quick and simpleand a PC.

We will study the system to obtain inputloutput data, to do this we must monitor and collect; initially we will try to give a specific time for each reading. For example, we collect data every two minutes or every five minutes and so on.
Another important thing is the selection a valid data format that can be processed with a computer. Finally, we should note and monitor the external factors which may effect on the validation of reading data.

\section{A. XBee Configuration}

To determine the type of unit used in this case we use the $\mathrm{X}$-CTU software which determines the type of XBee that we need to use it (Coordinator, End Devices).

\section{B. XBee Types}

In this work we classified the XBee in totwo types:

1. Coordinator:is the center of the system,It collects sensor readings and back it to the PC (user)[20].The flowchart of the coordinator is showing in figure 8 .

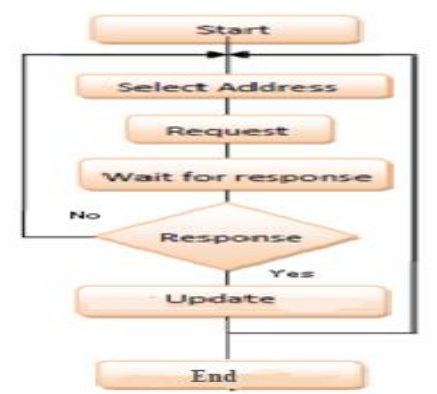

Fig. 8 flowchart of the coordinator

2. End Device: each end device can connect one or more temperature sensor input; the coordinator read the data request and the end device response to the request with the value from the sensor. The End Device interface consists of two parts: Sensor Interface and Xbee Interface, These two parts work in USB Board, Xbee Shield and TMP36 [20].The flowchart of the end device is shown in figure 9 .

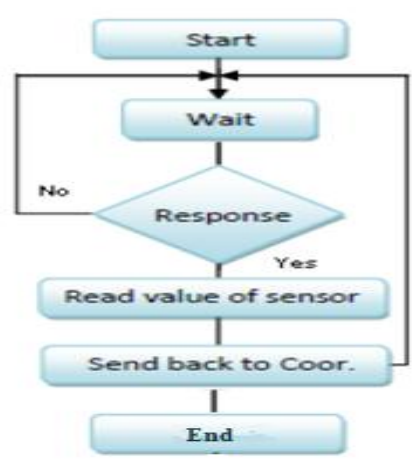

Fig.9 flowchart of the end device

We will need two XBee the first one is connect to PC through the USB which is a coordinator and the other one is an end device (Router) which is connected to the TMP36 where it transfer the signal wirelessly to the coordinator, each XBee has its own information (address, version, operating ID) so that we can reach it, we will use the XBee hardware in the matlab programming to access each XBee depending on its information. 
Vol. 4, Issue 2, February 2017

\section{Connect the XBee with Temperature Sensor}

We connect the temperature sensor with the XBee In the room that we want to be senses its temperature by using a Protoboard, we use the XBee breakout board as a template model place into the Protoboard as shown in figure 10, then connect the temperature sensor to the protoboard using telephone wire.

And we will supply with power $2 \times \mathrm{C}$-batteries each in figure 11 see the final product after connect all parts.

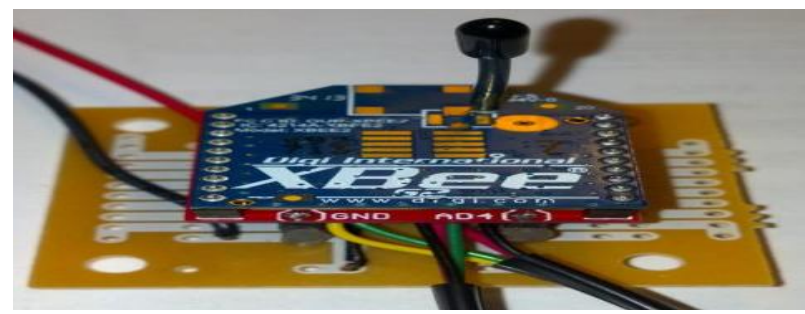

Fig. 10 XBee placed into the protoboard

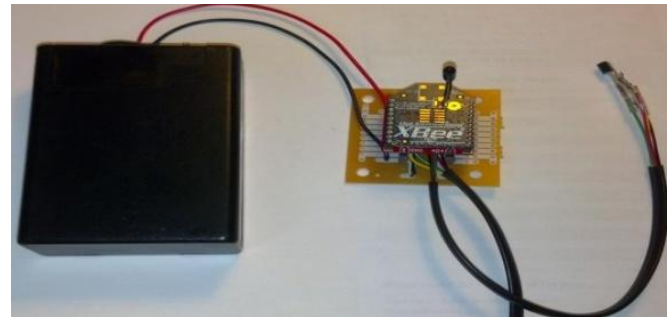

Fig. 11 connect the XBee with temperature sensor

\section{D.Connect the XBee with the PC}

We connect the XBee (coordinator) to PC using the USB cable as show in Fig. 12.

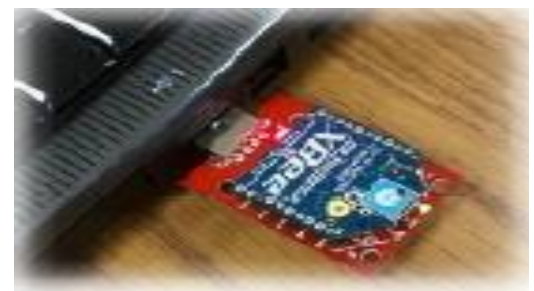

Fig.12 Connect the XBee with the PC

\section{E. Collect Data from Temperature Sensor}

After it has been put the temperature sensor in the room and connect it to the XBee we want to read the current temperature in the room and write the data to a file so that we could analyze it.

As we said previously we must open a connection from MATLAB to the XBee coordinator.

Then the voltage request from each pin on the XBee and record the answers. Then the voltage values are placed in an external file (log file) to store the values that we need it. To calculate and gather the data periodicallyaccording to a specific time it is done using a MATLAB timer.

The data will store in an external file, Data can be placed and arranged in the form of a table in MATLAB this will done using the read table function.

data=readtable('logfile.txt','Delimiter','It','ReadVariableNa mes',false);
Then to make sure that the read function work the plot function will used in matlab to draw the raw voltage data to the specific time as shown in figure 13 .

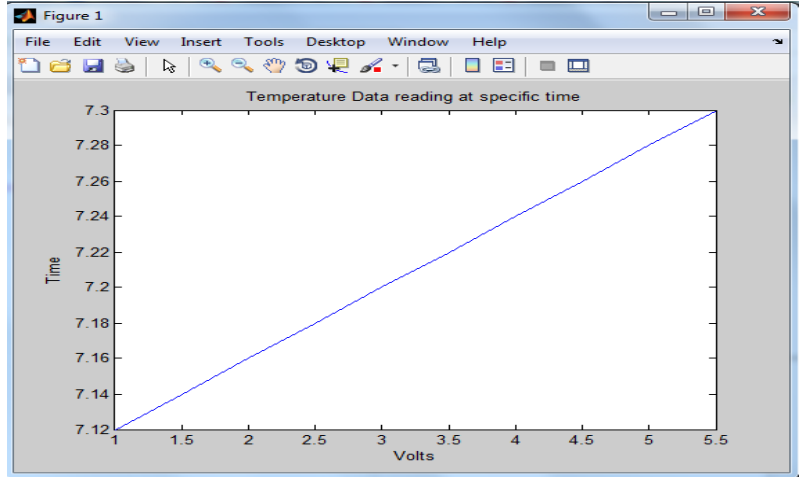

Fig. 13 The raw voltage data to the time

Then the voltage will change to Celsius degree using the function:

tempC $=($ volts $* 100-50)$;

After convert the voltage into temperature (Celsius degree), it is easily to plotted the temperature data to a specific time as shown in figure 14 .

plot(ts,tempC);

xlabel('Time');

ylabel('Temperature (IcircC)'); title(' Temperature Data reading');

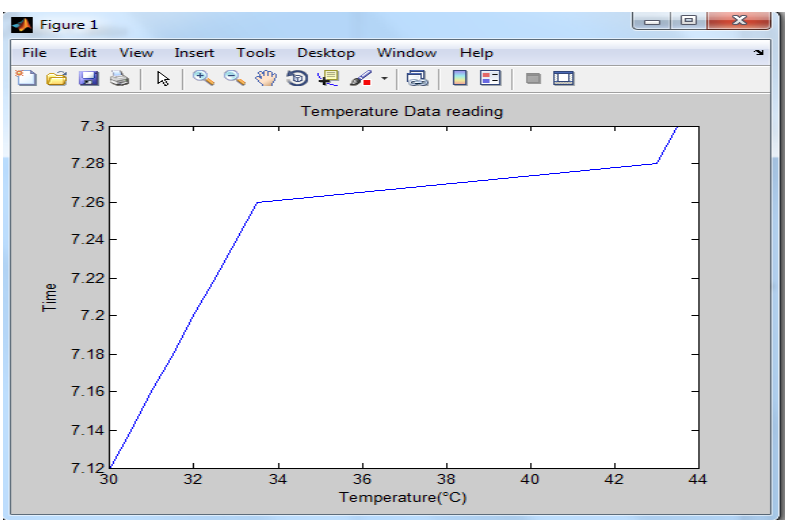

Fig .14 the reading temperature data at a specific time

\section{F. The Output Analysis}

When the data is displayed as required we can analyzed it in order to understand the system behavior, these data are used to get information about the behavior of the original system, And these data give us an idea about the approximate rate of temperature during the whole day, so for this we will have an idea of degrees of heat for each place we want to know its temperature.

\section{CONCLUSION}

The wireless sensor system is considered one of the most modern important techniques used in many fields in a life, because of its importance these systems are actively monitor their surrounding environment, it is easily to conclude and deduct other information from their data. 
Vol. 4, Issue 2, February 2017

The points summarize the main conclusions in this project:

1. This project highlighted the advantage of using special types of temperature sensor (TMP36) and its usage in sensing function.

2. When the sensor sense the temperature, it transfer the signals wirelessly using the XBee device.

3. The XBee (coordinator) receive the signal and send it to the PC that are associated with it.

4. it focuses on how to transfer data wirelessly and take advantage of that data letter.

\section{ACKNOWLEDGMENT}

I would like to express my deep gratitude to DR. Ashwaq.Q.Hameed for her supervision during the period of the research, encouragement, comment and help. And I would like to thank my family, and my close friends who stand beside me in my study and gave me encourage, support and help to complete my study.

\section{REFERENCES}

[1] M. Bokare and M. A. Ralegaonkar, "Wireless sensor network: A promising approach for distributed sensing tasks," Excel Journal of Engineering Technology and Management Science, vol. 1, pp. 1-9, 2012.

[2] A. Zografos, "Wireless Sensor-based Agricultural Monitoring System," 2014..

[3] P. CAST, "Lifetime Power® Wireless Sensor System," Excel Journal of Engineering Technology and Management Science, JAN 302012 ..

[4] Dr. Jose Garcia Lecture 2 MMAE 415: Aerospace Laboratory II Spring 2012.

[5] R. Faludi, "Building Wireless Sensor Networksâ€ $\square$, Oâ€тMReilly Media," Inc, 2011.

[6] F. M. Mart ̃̂n, "sensors," arXiv preprint arXiv:1305.1259, vol. 232, pp. 26,27, 2011..

[7] J. Yick, B. Mukherjee, and D. Ghosal, "Wireless sensor network survey," Computer networks, vol. 52, pp. 2292-2330, 2014.

[8] I. Rekleitis, "Introduction to Robotics and intelligent system " Journal of Systems and Software, 2013.

[9] L. Mainetti, L. Patrono, and A. Vilei, "Internet of Things:Wireless Sensor Networks: A survey," in Software, Telecommunications and Computer Networks (SoftCOM), 2011 19th International Conference on, pp. 1-6.

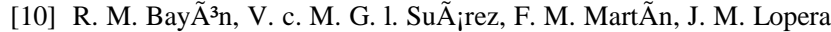
Ronda, and J. C. Ã. 1. Ant $\tilde{A}^{3} n$, "A Wireless Portable High Temperature Data Monitor for Tunnel Ovens," Sensors, vol. 14, pp. $14712-14731$.

[11] A. R. Center, "SENSORS: Types and Characteristics," Excel Journal Engineering Technology and Management Science, vol. 27, 2013.

[12] P. S. NAEEM, "Characterization \& Optimization of Temperature Sensor Using LABVIEW," in Software, Telecommunications and Computer Networks (SoftCOM), p. 88, 2014

[13] Temperature Sensor Design Guide, Temperature Measurement Solutions for Silicon IC Temperature Sensor, Thermocouple, RTD and Thermistor-Based Applications.

[14] ada, "TMP36 Temperature Sensor," pp. 5-6, 2016-sep.

[15] Hans--Petter, H. Telemark, University, College, Faculty, of, Technology, Department, of, Electrical, Engineering, InformaDon, Technology, and, and CyberneDcs, "TMP36 Temperature Sensor," Excel Journal of Engineering Technology and Management Science, 2015.

[16] Hans--Petter and Halvorsen, "XBee Wireless Communication," 2014.

[17] Hans--Petter and Halvorsen, "XBee Wireless Communication," 2014.
[18] V. Boonsawat, J. Ekchamanonta, K. Bumrungkhet, and S. Kittipiyakul, "XBee wireless sensor networks for temperature monitoring," in the second conference on application research and development (ECTI-CARD 2010), Chon Buri, Thailand.

[19] E. Chobot, D. Newby, R. Chandler, N. Abu-Mulaweh, C. Chen, and C. Pomalaza-RÃ $\tilde{A}_{i} \mathrm{z}$, "Design and Implementation of a Wireless Sensor and Actuator Network for Energy Measurement and Control at Home," arXiv preprint arXiv:1305.1259, 2013.

[20] "XCTU GUIDE XCTU Configuration and Test Utility Software," October 2015. 